\title{
Das gallorömische Theater von Dalheim (Großherzogtum Luxemburg)
}

\section{Peter Henrich}

\section{(2) OpenEdition}

\section{Journals}

Édition électronique

URL : http://journals.openedition.org/edl/109

DOI : 10.4000/edl. 109

ISSN : 2296-5084

\section{Éditeur}

Université de Lausanne

\section{Édition imprimée}

Date de publication : 15 mai 2011

Pagination : 129-152

ISBN : 978-2-940331-25-3

ISSN : 0014-2026

\section{Référence électronique}

Peter Henrich, « Das gallorömische Theater von Dalheim (Großherzogtum Luxemburg) », Études de lettres [Online], 1-2 | 2011, Online erschienen am: 15 Mai 2014, abgerufen am 19 Dezember 2020. URL http://journals.openedition.org/edl/109; DOI : https://doi.org/10.4000/edl.109 


\section{DAS GALLORÖMISCHE THEATER VON DALHEIM (GROSSHERZOGTUM LUXEMBURG)}

Bei den Grabungen im gallorömischen Theater von Dalheim konnten neben zwei aufeinander folgenden hölzernen Sitzbankkonstruktionen in der gesamten cavea das Einbringen von Sitzreihen aus Kalkstein sowie einer zusätzlichen Sitzreihe aus Sandstein in der orchestra dokumentiert werden. Im Rahmen des Umbaus der Sitzreihen wurde die cavea-Mauer bis auf das Fundamentniveau abgerissen. Beim anschließenden Wiederaufbau verstärkte man zusätzlich die Ecken des Theaters und mauerte die äußeren Apsiden zu. Diese Umbauten sind als eine präventive Maßnahme und nicht als Reparatur bzw. als Reaktion auf bereits aufgetretene Schäden im Mauerwerk zu werten. Die steinernen Sitze in den ersten beiden Reihen mit Rücken- und auch Armlehnen sind als deutliche Hinweise auf die Prohedrie in einem gallorömischen Theater zu deuten. Die Trittstufen auf die Bühne, die seitlichen Eingänge neben dem Bühnengebäude sowie die Altäre in der orchestra liefern wichtige Hinweise zur Funktion der gallorömischen Theater. Die Grabungen haben eine Nutzung des Geländes vor dem Theaterbau im ersten Viertel des 2. Jahrhunderts als Steinbruch belegt. Außerdem wurde ein dort stehendes Gebäude beim Bau des Theaters abgerissen. Nach den Germaneneinfällen des 3. Jahrhunderts wird ein Teil der Sitzsteine für den Bau eines Burgus genutzt und das Theater verliert seine ursprüngliche Funktion. Eine funktional bislang nicht näher bestimmbare Nutzung ist bis an das Ende des 4. Jahrhunderts aufgrund von Kleinfunden belegt. 


\section{Forschungsgeschichte und Lage des Theaters}

Nach den ersten Grabungskampagnen 1985 und 1999 bis $2003^{1}$ wurden die Forschungen im gallorömischen Theater von Dalheim sowie in dessen direktem Umfeld in den Jahren 2007 und 2008 im Auftrag des Musée National d'Histoire et d'Art unter Leitung des Verfassers fortgeführt. Hierbei konnten zentrale Fragen zur Datierung des Theaters sowie zu Umfang und Abfolge der Umbauten im Theater beantwortet werden. Zudem sind nun erstmals detaillierte Aussagen zur Struktur und Genese des direkten Außenbereiches möglich.

Der auf der Tabula Peutingeriana eingezeichnete römische vicus RICCIACO bei Dalheim befindet sich auf dem höchsten Punkt des Pëtzel-Plateaus im Luxemburger Sandsteingebiet zwischen den Dörfern Dalheim und Filsdorf auf einer Höhe von 310-325 m über NN. Das heute fast ausschließlich ackerbaulich genutzte Gelände fällt nach Südwesten hin sanft ab und ist im Norden durch einen felsigen Steilhang begrenzt. Lange Zeit ging man davon aus, dass sich der vicus vor allem auf dem Pëtzel-Plateau erstreckte. Nach der Entdeckung des Theaters 1985 sowie eines Thermengebäudes ${ }^{2}$ in den Jahren 2003 und 2004 im Dorfkern wird nun deutlich, dass sich unter der rezenten Bebauung weitere, vermutlich öffentliche, Bauten des römischen vicus befinden.

Das Theater (Abb. 1) lehnt sich an den bereits beschriebenen ost-west verlaufenden und nach Norden hin exponierten felsigen Steilhang. Die Frontfassade ist nordnordwest-ostostsüd ausgerichtet und weicht von den durch die römischen Fernstraßen vorgegebenen Baufluchten auf dem Pëtzel-Plateau ab (Abb. 2).

Durch umfangreiche Erosionen seit dem vierten Jahrhundert sind die Sitzsteine der ersten acht Ränge, zahlreiche weitere Befunde in der orchestra sowie die noch bis in eine Höhe von $2 \mathrm{~m}$ dokumentierte Frontmauer und große Teile der rückwärtigen cavea-Mauer zusedimentiert worden

I. J. Krier, R. Wagner, „Das Theater des römischen Vicus in Dalheim “ und „Das römische Theater in Dalheim “; J. Krier, „Le théâtre gallo-romain découvert en 1985 à Dalheim “; R. Wagner, „Théâtre gallo-romain de Dalheim “; J. Krier, „Agglomération romaine de Dalheim-Ricciacum ".

2. J. Krier, „Das Thermengebäude des römischen Vicus von Dalheim?“. 


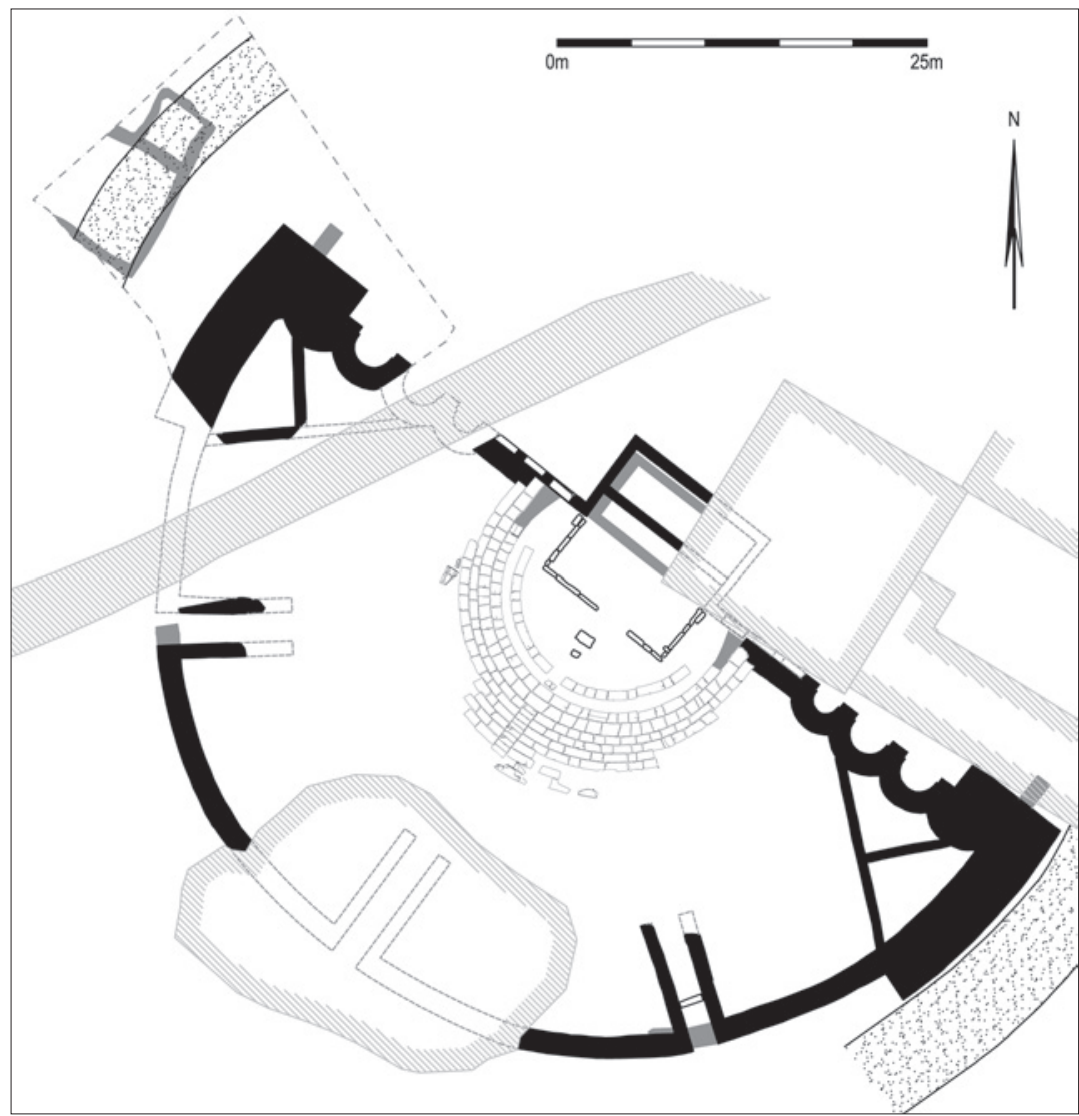

Abb. 1 - Gesamtplan des Theaters von Dalheim.

und sehr gut erhalten. Lediglich ein geringer Abschnitt der rückwärtigen cavea-Mauer ist durch einen rezenten Steinbruch zerstört.

\section{Vortheaterzeitliche Nutzung und Bebauung des Areals}

Im nordwestlichen Außenbereich, ca. $5 \mathrm{~m}$ von der Theatermauer entfernt, befand sich ein Gebäude, das unmittelbar vor dem Bau des Theaters abgerissen wurde und von dem sich lediglich die Ausbruchsgräben der Fundamente erhalten haben. Das $12 \mathrm{~m}$ tiefe Gebäude konnte auf einer Länge von $7 \mathrm{~m}$ dokumentiert werden und 


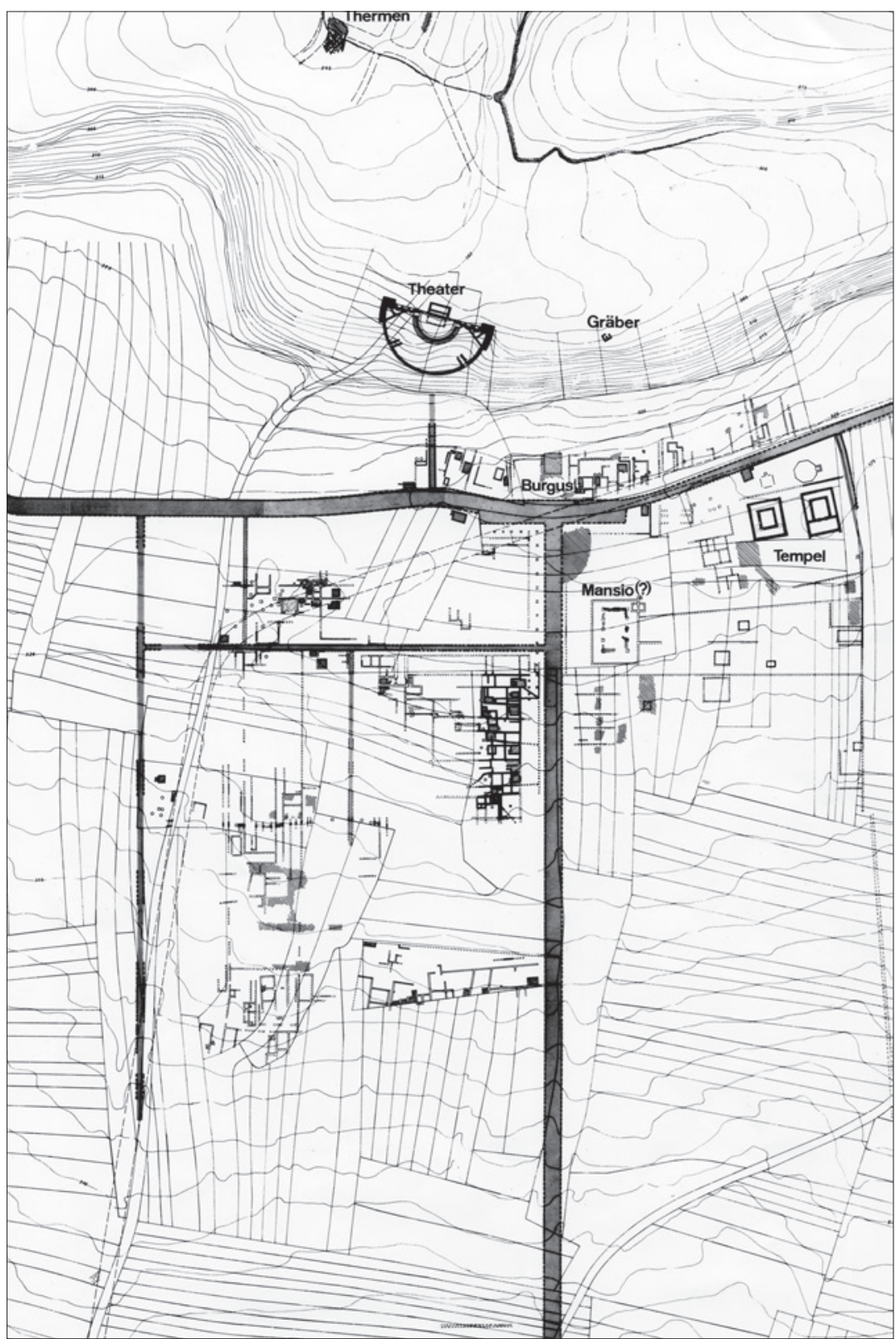

Abb. 2 - Plan des römischen vicus Ricciaco/Dalheim. 
bestand aus einem Raum mit $7 \mathrm{~m}$ lichter Breite, an den risalitartig ein kleiner Raum angebaut war. Der nördliche Abschluss des Hauses liegt außerhalb der Grabungsfläche - in Analogie zu den unten angeführten Parallelen ist dort ein zweiter kleiner Risalit zu rekonstruieren. Ein Bodenbelag war nicht nachzuweisen. Mit Ausnahme eines unspezifischen Ofens fehlten im Inneren Befunde, die Rückschlüsse auf die Funktion des Gebäudes erlauben. Parallelen für diesen Gebäudetyp finden sich in Haupt- jedoch vor allem in Nebengebäuden großer gallorömischer villae rusticae. Diese Gebäude werden in der Regel als Speichergebäude oder Scheunen interpretiert ${ }^{3}$. Doch auch in vici ist diese, keiner bestimmten Funktion zuzuordnende Gebäudeform anzutreffen, wie die Beispiele aus Bliesbrück ${ }^{4}$ oder in langrechteckiger Form aus Dampierre-Fontenelle ${ }^{5}$ zeigen. Durch die genannten Parallelen lässt sich eine Frontlänge von mindestens $13,50 \mathrm{~m}$ rekonstruieren.

Neben einzelnen, direkt neben Theatern zeitgleich existierenden Gebäuden ${ }^{6}$, ist der Abriss von Bauten zum Bau eines Theaters eine überregional erkennbare Entwicklung, wie die Beispiele von Arleuf ${ }^{7}$ und Argentomagus zeigen ${ }^{8}$. Nicht näher beschriebene Funde und Befunde im Bereich der Theater von Alésia, Vendeuil-Caply und Neung-sur-Beuvron belegen auch dort eine Nutzung des Geländes vor dem Bau der Theater ${ }^{9}$. Im südwestlichen und südlichen Theaterumfeld fanden sich eindeutige Hinweise auf eine intensive Nutzung als Steinbruch. Dies belegen Abschrotungsspuren von Steinblöcken sowie mehr als $4 \mathrm{~m}$ tiefe treppenartige Abarbeitungen des Felses südlich des Südvomitoriums (Abb. 3).

3. Ch. Gaston, „Bâtiments, standardisés ' dans la pars rustica des villae“.

4. J.-P. Petit, „L'architecture privée dans l'agglomération secondaire de Bliesbruck“, S. 130, Abb. 2.

5. J.-P. Petit, M. Mangin, P. Brunella, Atlas des agglomérations secondaires de la Gaule Belgique et des Germanies, S. 23, Abb. 7.

6. So z.B. in Compierre (M. Bonneau, „Le théâtre de Compierre “, S. 133, Abb. 4) und Verulamium (K. M. Kenyon, „The Roman theatre at Verulamium, St. Albans“, Taf. 69-70).

7. L. Olivier, „Arleuf“ und „Le théâtre antique de Bardiaux “, S. 60, Abb. 1; A. Olivier, „Grand“.

8. F. Dumasy, Le théâtre d'Argentomagus, S. 45 sq.

9. Alésia: A. Olivier, E. Rabeisen, „Le théâtre d’Alésia “, S. 60; Vendeuil-Caply: G. Dufour, „Vendeuil-Caply “, S. 70; Neung-sur-Beuvron: H. Delétang, „Le théâtre gallo-romain de Neung-sur-Beuvron ", S. 176. 


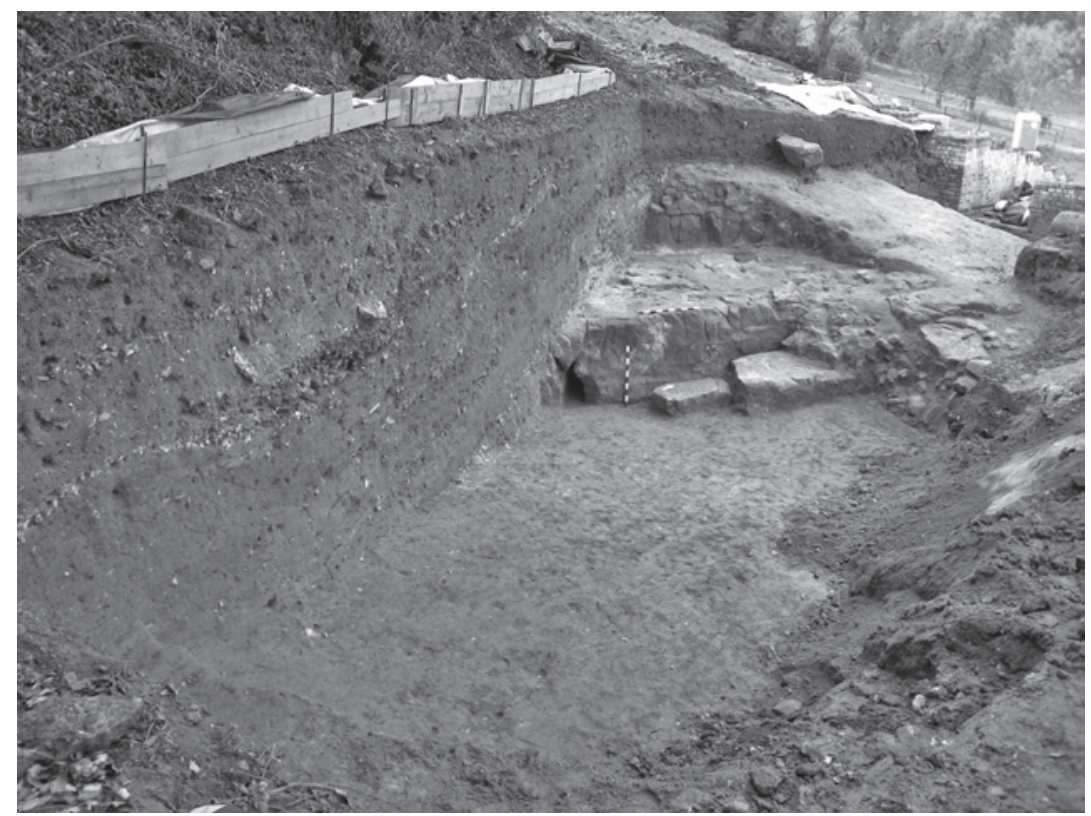

Abb. 3 - Sondage südlich des Südvomitoriums. Deutlich sind die treppenartigen Abarbeitungen im anstehenden Fels sowie die mächtigen Verfüll- und Planierschichten zu erkennen.

\section{Das erste Theater}

In den Jahren 2007 und 2008 wurden im Theaterinnenraum Reste des Unterbaus für hölzerne Sitzbänke in Form von Abarbeitungen im anstehenden Fels (Abb. 4) sowie Pfosten- und Balkengruben in dem aufgefüllten Sediment dokumentiert. Wie auch bei den Theatern von Blicquy ${ }^{10}$ und Verulamium $^{11}$ ist für das erste Theater von einer steinernen caveaMauer und Sitzreihen aus Holz auszugehen.

Anhand der Stratigraphie war zweifelsfrei zu erkennen, dass die Bereiche von ima cavea und media cavea einerseits aus dem Fels herausgearbeitet waren und in anderen Bereichen in großem Umfang dort Sediment aufplaniert wurde, wo bedingt durch die Steinbruchtätigkeit der Fels fehlte. Das dokumentierte Profil zeigte eindeutig, dass die summa cavea in der ersten Bauphase des Theaters nicht mit Sediment

Io. E. Gillet, L. Demarez, D. Deroissart, „Leuze-en-Hainaut/Blicquy“.

II. K. M. Kenyon, „The Roman theatre at Verulamium, St. Alban “, S. 218. 


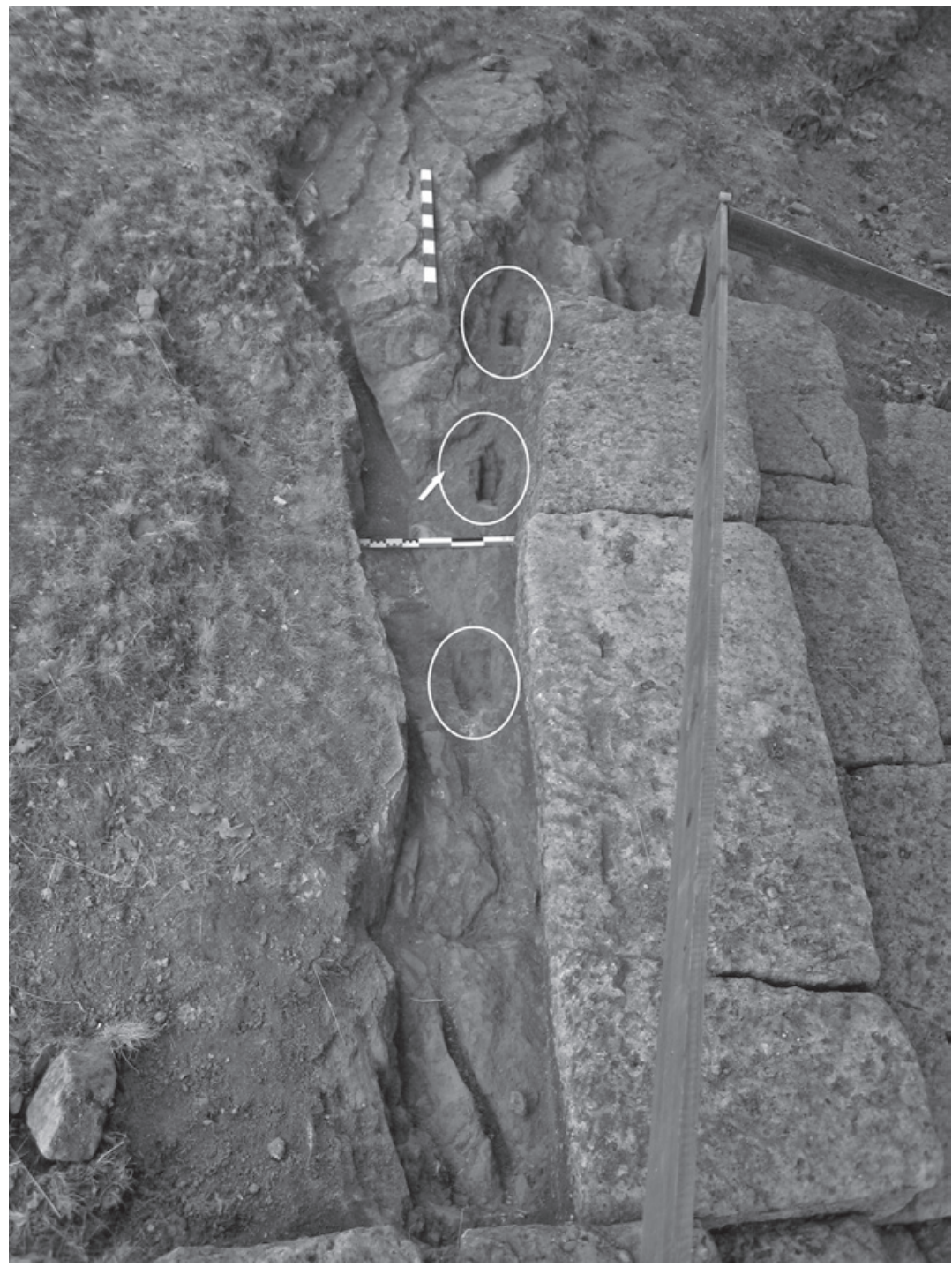

Abb. 4 - Pfostenlöcher (Kreismarkierung) als Stabilisierung der ersten Holzbänke im Theater im anstehenden Fels direkt hinter der letzten erhaltenen Sitzreihe aus Kalkstein. 

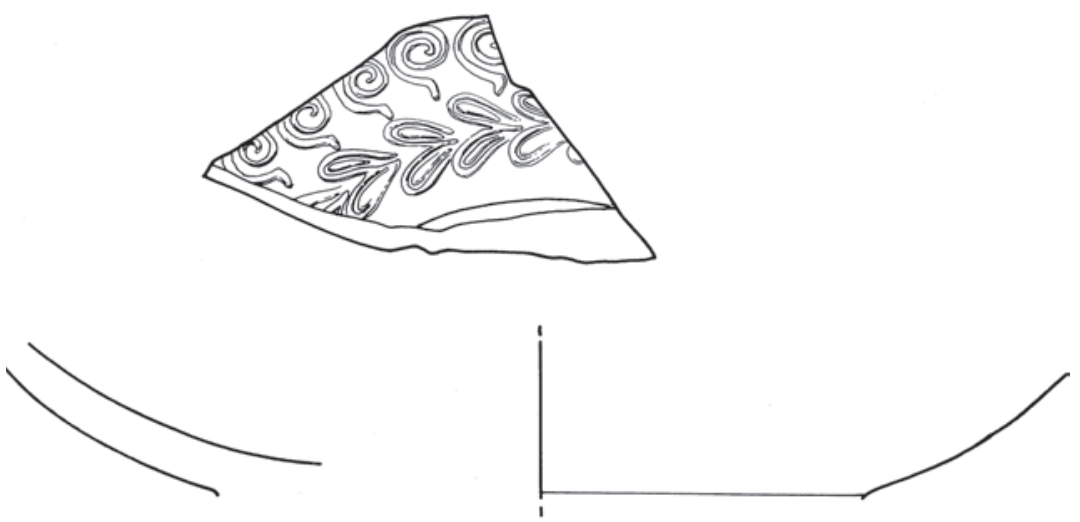

Abb. 5 - Auf dem anstehenden Boden unter der untersten Auffüllschicht gefundene Wandscherbe einer Drag. 37 Schüssel Trierer Produktion.

aufgefüllt war und die Sitzbänke hier auf einem freistehenden Gerüst zu rekonstruieren sind.

Die durch die vorherige Nutzung des Areals als Steinbruch notwendigen massiven Auffüllungen vor allem im Bereich der Ecken erklären die den Hangdruck abfangenden und die Erdmassen stabilisierenden halbrunden Apsiden in der Frontmauer, die für gallorömische Theater in dieser Form mit wenigen Ausnahmen ungewöhnlich sind ${ }^{12}$. Aus diesen Planierschichten stammen nur sehr wenige kleinteilige Funde, was gegen eine Auffüllung mit Sediment aus dem unmittelbaren Siedlungsumfeld spricht. Dies wäre ansonsten, wie das Beispiel des Theaters von Alésia zeigt ${ }^{13}$, stark mit Siedlungsabfall wie Keramik- und Tierknochenfragmenten durchsetzt. Somit muss es sich um Sediment handeln, das bei Bau- oder Steinbrucharbeiten in der Nähe angefallen ist.

Bislang ist nichts über die Eingänge des ersten Dalheimer Theaters bekannt. Eventuell gelangte man über seitlich an die Außenseite der rückwärtigen cavea-Mauer angebaute L-förmige Treppenaufgänge ${ }^{14}$ in

I2. Saint-Cybardeaux: M. Fincker, F. Thierry, „Nouvelles recherches sur le théâtre gallo-romain des Bouchauds (Charente) “; Autun: A. Olivier, „Autun “, S. 38 sq.; Soissons: F. Dumasy, „Petit atlas des édifices de théâtres en Gaule romaine “, S. 73.

13. A. Olivier, E. Rabeisen, „Le théâtre d'Alésia “, S. 62 sq.

I4. So z.B. in Alesia: A. Olivier, E. Rabeisen, „Le théâtre d'Alésia “, S. 60 ; Saint Cybardeaux: C. de La Croix, „Etude sur le théâtre gallo-romain des Bouchauds (Charente) et son déblaiement “, Taf. 2 P und Q; M. Fincker, F. Thierry, „Nouvelles 
das Theater. Letztendlich beweisen lässt sich dies im Dalheimer Theater nicht, da die rückwärtige cavea-Mauer bis in die Fundamentbereiche in den nachfolgenden Bauphasen sehr stark verändert wurde und sich keine Spuren erhalten haben.

Der Bau des ersten Theaters kann aufgrund einer unter der untersten Planierschicht und auf dem anstehenden Sediment in der media cavea gefundenen Drag. 37-Wandscherbe aus Trierer Produktion (Abb. 5) frühestens in das erste Viertel des zweiten Jahrhunderts nach Christus datiert werden ${ }^{15}$.

Diese Datierung wird durch das Fundmaterial aus den jüngsten Siedlungsschichten des oben beschriebenen Gebäudes neben dem Theater bestätigt.

\section{Umbauten im Theater}

Bei den Grabungen konnten mehrere Bauphasen dokumentiert werden. Trotzdem änderten sich weder Orientierung noch Grundriss des Theaters grundlegend ${ }^{16}$.

So wurde beispielsweise im Bühnengebäude die Außenmauer der ersten Bauphase abgerissen und durch eine neue Mauer unmittelbar daneben ersetzt (Abb. 6). Der Grund für diese Maßnahme ist nicht bekannt.

Umfangreiche Änderungen konnten an der Innenseite der 2007 vollständig freigelegten rückwärtigen cavea-Mauer östlich des Südvomitoriums dokumentiert werden. Hier war der Abriss bis auf das Fundamentniveau und der komplette Wiederaufbau der Mauer in römischer Zeit deutlich zu erkennen (Abb. 7). Bei dem Wiederaufbau verstärkte man zusätzlich die Ecken des Theaters und mauerte die äußeren Apsiden zu. An diese Mauer wurden in einer späteren Bauphase die Vomitorien gebaut. Die Profile im Theaterinnenraum zeigen, dass der

recherches sur le théâtre gallo-romain des Bouchauds (Charente) “, S. 120 sq., S. 132, Abb. 26 und S. 133, Abb. 27; Argentomagus: F. Dumasy, Le théâtre d'Argentomagus (Saint-Marcel, Indres), S. 127 sq.; Moingt: J. Renaud, „Le théâtre mixte d'Aquae Segetae“, S. 289, Abb. 3.

I5. I. Huld-Zetsche, Trierer Reliefsigillata, S. 78. Bei dem Dekor handelt es sich um das gefüllte Doppelblatt O49 sowie die Hakenspirale O33a.

I6. Vgl. hierzu die Ausführungen von F. Dumasy, „Les édifices de spectacle en Gaule du Nord “, zur chronologischen Entwicklung der Theater anhand des Grundrisses. 


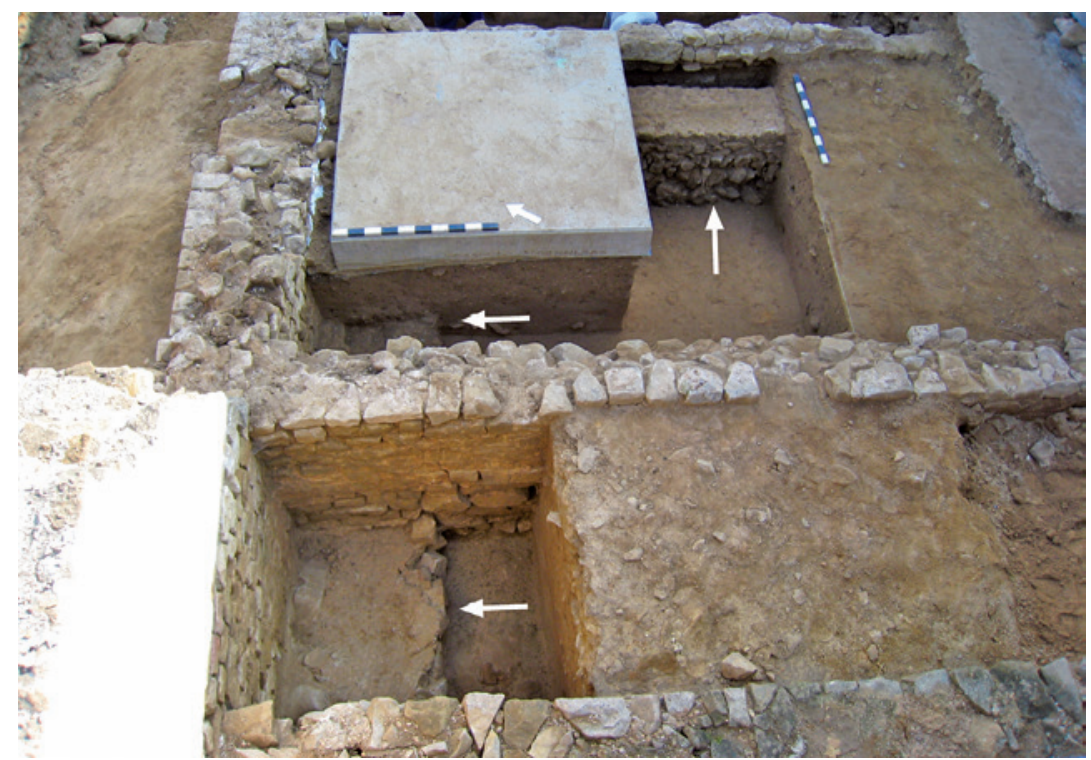

Abb. 6 - Blick von der orchestra in das Bühnengebäude mit den Mauern der ersten (Pfeile) sowie der jüngsten Bauphase.

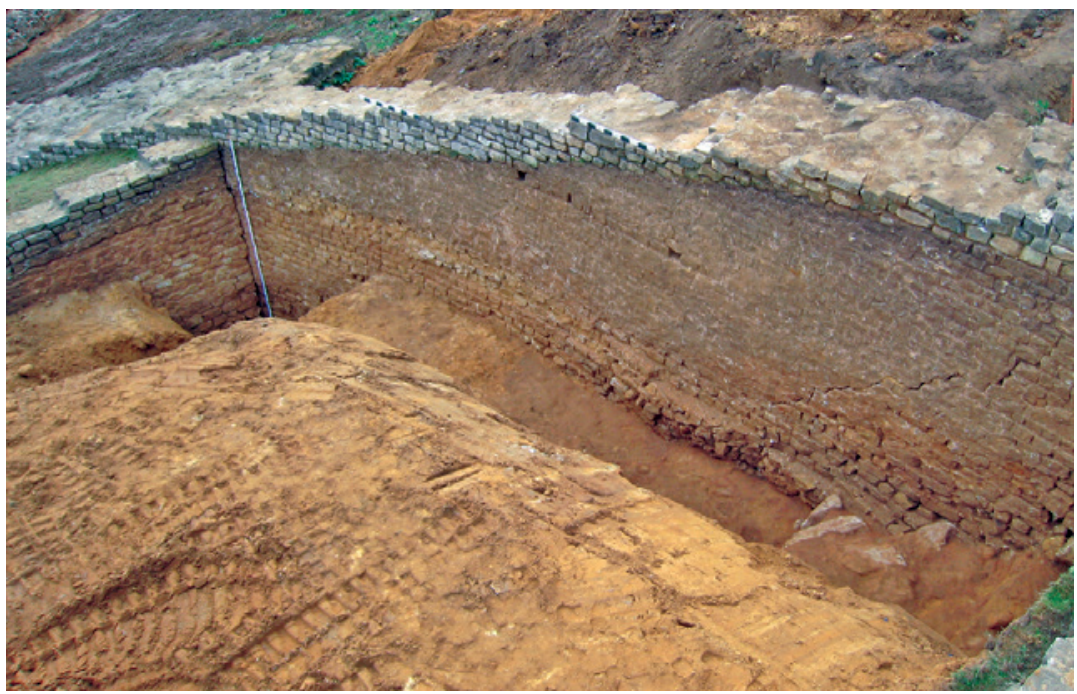

Abb. 7 - Innenseite der südlichen Theateraußenmauer mit Hinweisen auf einen Abriss und Neubau der Mauer auf Höhe des Fundamentes. Erkennbar ist ein Wechsel im Baumaterial. 


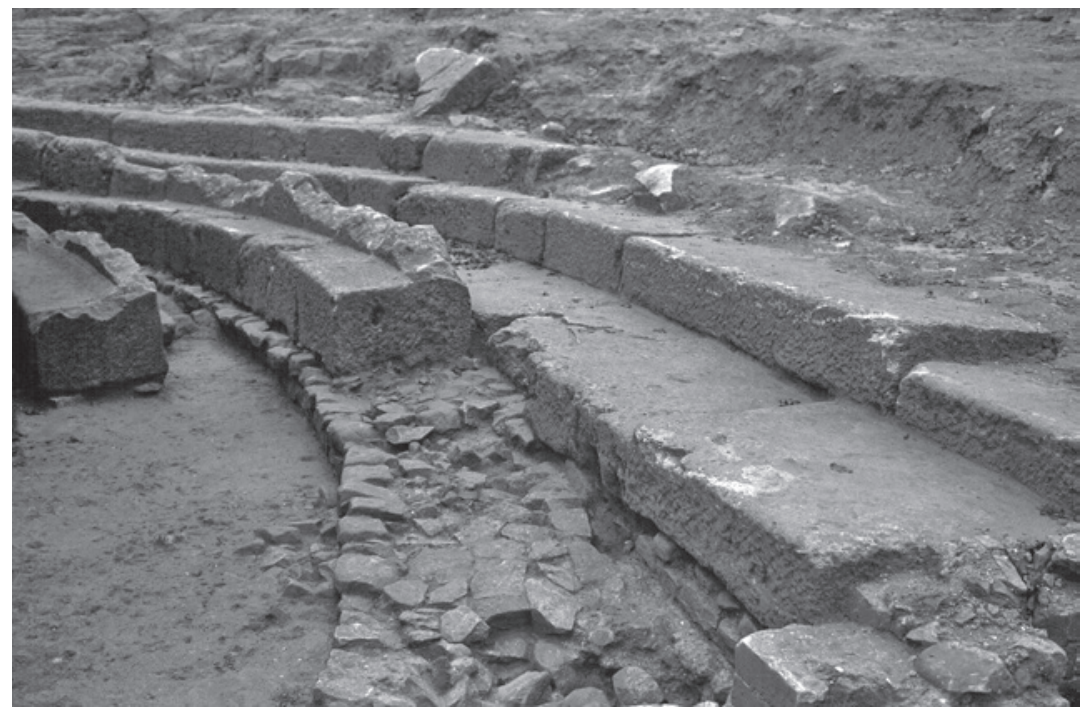

Abb. 8 - Blick vom Eingang nördlich des Bühnengebäudes auf die unter der Sitzreihe noch vorhandene Stützmauer, die den Hangdruck des Sedimentes in der cavea vor dem Einbringen der steinernen Sitzreihen abfangen sollte.

Umbau im Vorfeld einer weiteren Sedimentaufschüttung in der summa cavea erfolgte. Demnach handelt es sich bei dem Abriss und der zusätzlichen Verstärkung der Theaterecken um eine präventive Maßnahme und nicht um eine Reparatur bzw. eine Reaktion auf bereits aufgetretene Schäden im Mauerwerk.

Bei den Grabungen 2007 wurde deutlich, dass die quer in den Theaterecken verlaufenden Mauern bereits zur ersten Bauphase gehörten und nicht nachträglich eingebaut wurden. Somit muss bereits beim Bau des Theaters der große Druck auf die Außenmauern und hier besonders die Ecken bekannt gewesen sein. Insofern ist auch bei anderen Theatern in Gallien zu prüfen, ob vergleichbare Befunde, die bislang als Reparaturmaßnahme und Reaktion auf entstandene Bauschäden gedeutet wurden, nicht im Kontext eines Umbaus beispielsweise bei der Ausstattung mit steinernen Sitzreihen zu sehen sind.

Wie anhand mehrerer in dieser Sedimentauffüllung dokumentierter Pfostengruben deutlich wird, sind im Dalheimer Theater vor dem Einbringen der Sitzreihen aus Stein eine zweites Mal hölzerne Sitzreihen errichtet worden. Als Sicherungsmaßnahme im Rahmen dieser Aufhöhung des Sedimentes ist auch der Bau einer die orchestra 


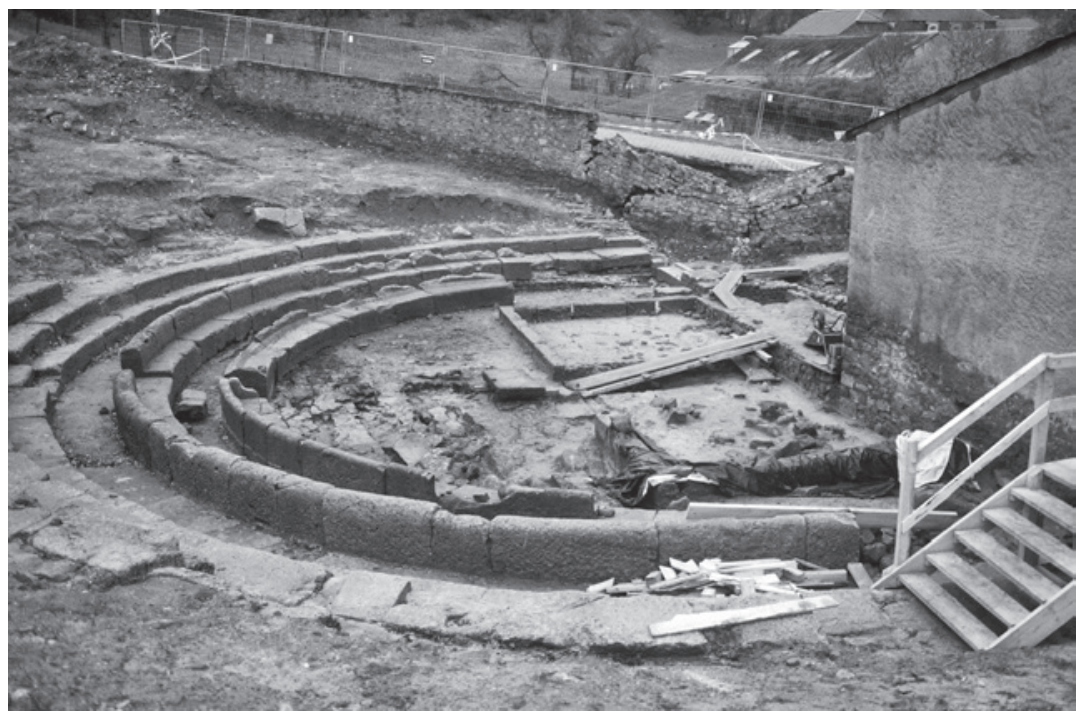

Abb. 9 - Bis auf den anstehenden Fels freigelegter Theaterinnenraum während der Grabungen im Jahr 1999.

begrenzenden über das Halbrund hinausgehenden Mauer zu sehen, die die Funktion hatte, den nun größeren Hangdruck in Richtung orchestra abzufangen (Abb. 8). Die Baufuge zur Innenseite der Frontmauer und der Abriss beim Einbringen der steinernen Sitzreihen bei den nachfolgenden Umbauten sind eindeutige Hinweise auf die Zuordnung in diese Bauphase des Theaters.

In der darauf folgenden Bauphase wurden die Sitzreihen aus Kalkstein eingebaut, von denen heute noch die Reihen der ima cavea erhalten sind (Abb. 9). Aufgrund der Erhaltungsbedingungen ist nicht zu entscheiden, ob das gesamte Theater mit steinernen Sitzreihen ausgestattet war oder nur die untersten Reihen. Einen indirekten Hinweis liefert der Befund des im 19. Jahrhundert ausgegrabenen burgus, der fast ausschließlich aus Kalksteinblöcken des Theaters errichtet wurde ${ }^{17}$. Rechnet man diese Steine zu den noch erhaltenen hinzu, so sind für das gesamte Theater steinerne Sitzreihen zu postulieren.

Der identische Kalkstein der Sitzsteine und der Schwellen in den Vomitorien sowie in den Eingängen neben dem Bühnengebäude deutet gemeinsam mit den Baufugen zwischen den Vomitoriumswangen und

I7. J. Krier, „Agglomération romaine de Dalheim-Ricciacum “, S. 38. 
der rückwärtigen cavea-Mauer darauf hin, dass diese Eingänge erst beim Einbau der Sitzreihen aus Stein eingerichtet wurden.

Aus dem gleichen Kalkstein sind auch die linear angeordneten, bis zu 1,40 m langen und 0,38 $\mathrm{m}$ hohen, Fundamentblöcke der rechteckigen Bühne, die dem Bühnengebäude in der gesamten Breite von $10,10 \mathrm{~m}$ unmittelbar vorgelagert ist und 4,25 $\mathrm{m}$ in die orchestra hineinreicht (Abb. 9). Die Blöcke sind in den anstehenden Boden, auf dem Fels aufliegend, eingetieft.

Heute nicht mehr erhalten ist ein Kalksteinblock, der in Größe und Ausführung mit denjenigen des Bühnenfundamentes übereinstimmt und der in der Nordostecke der Bühne als Rest einer zweiten Steinlage gefunden wurde. Die Abarbeitung des Blockes zur Bühne hin diente als Auflage für Holzdielen ${ }^{18}$, und die Lage des Steines spricht dafür, dass sich auch auf den restlichen Fundamentsteinen der Bühne eine zweite Lage Blöcke befunden haben muss, die während des Steinraubes in der Spätantike entfernt wurde ${ }^{19}$. Direkt an der Bühne ist neben den Eingängen in der Frontmauer jeweils ein 0,44 m hoher und 0,80 m x $0,35 \mathrm{~m}$ großer, stark abgetretener Trittstein nachgewiesen, über den die Bühne von der orchestra aus betreten werden konnte.

Vor der ersten Sitzreihe aus Kalkstein, die mit einer Rückenlehne ausgestattet ist, platzierte man in einer der letzten Bauphasen eine zusätzliche Sitzreihe aus Sandstein mit den Rückenlehnen und Armlehnen an den Endblöcken (Abb. 10). Hiermit liegen zum ersten Mal in einem gallischen Theater gesicherte Belege für eine Prohedrie vor. Dies ist für andere gallorömische Theater zwar des Öfteren vermutet worden ${ }^{20}$, war jedoch aufgrund des in der Regel schlechten Erhaltungszustandes nicht sicher zu belegen.

Ebenfalls eine Besonderheit stellen die Fundamente von zwei Altären aus Sandstein in der orchestra dar (Abb. 11). Diese lagen in der Flucht der Treppe zwischen den Sitzreihen aus Sandstein und der Bühne. Von einem Altar war die in den Boden eingetiefte Fundamentplatte mit einer Grundfläche von $0,40 \mathrm{~m} \times 0,50 \mathrm{~m}$ sowie das einfach profilierte Unterteil

I8. Eine aus Holzdielen bestehende Bühne wird auch für das Theater von SaintGermain-d'Esteuil vermutet: M. Fincker, „Saint-Germain-d'Esteuil“, S. 173.

19. Folglich ist die heutige Rekonstruktion des Bretterbodens auf den Fundamentsteinen der Bühne im Vergleich zur antiken Situation zu niedrig.

20. So für das Theater von Argentomagus bei F. Dumasy, Le théatre d'Argentomagus (Saint-Marcel, Indres), S. 230. 


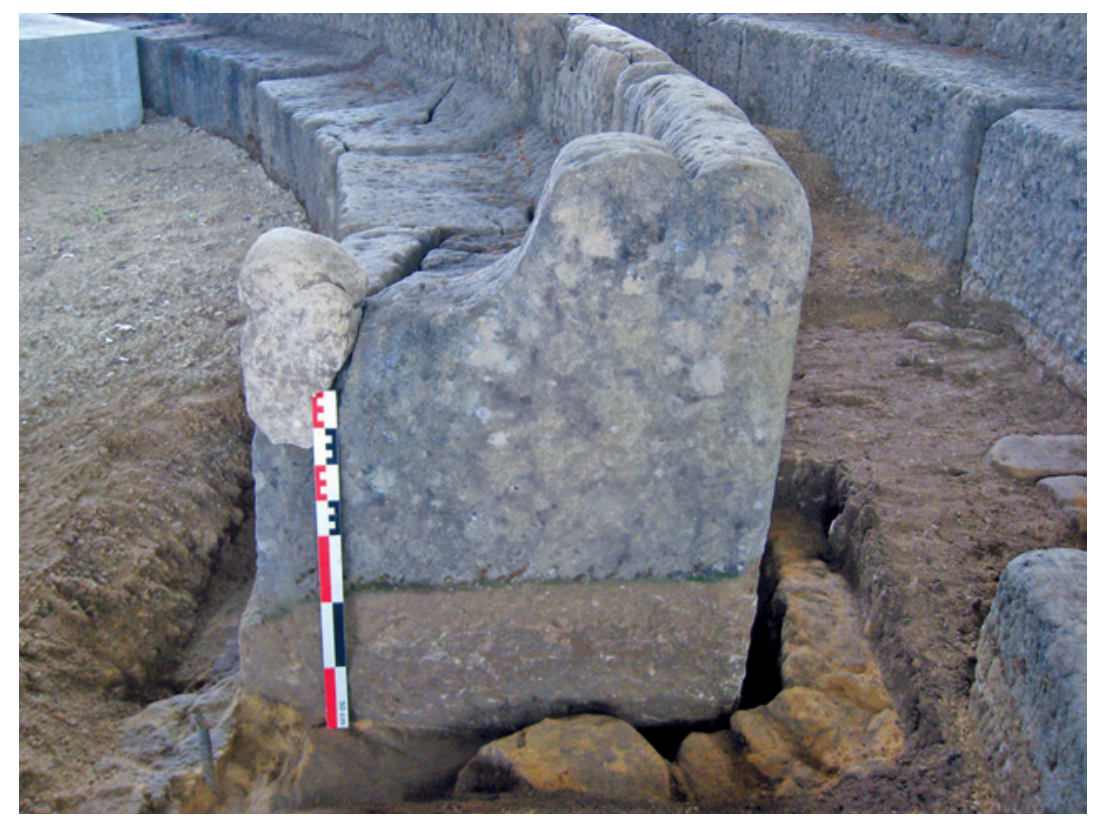

Abb. 10 - Nachträglich eingebaute Sitzreihe aus Sandstein mit Rücken- und Armlehne.

erhalten. Er befand sich in einem Abstand von 1,40 m von der Sitzreihe entfernt und mittig $0,60 \mathrm{~m}$ vor der max. $1,22 \mathrm{~m} \mathrm{x} 0,70 \mathrm{~m}$ großen, annähernd rechteckigen Fundamentplatte des zweiten Altares, die in 1,60 m Abstand von der Bühne gefunden wurde. Diese Fundamentplatte wies in der Mitte ein Loch zur Befestigung des Altarsteines auf.

Eine Parallele zu dem Dalheimer Befund gibt es bei den gallo-römischen Theatern lediglich in Vendeuil-Caply ${ }^{21}$. In Gallien ist ferner in Arles ${ }^{22}$ ein Altar in der Mitte der orchestra bekannt ${ }^{23}$. Hier geht Jules Formigé davon aus, dass dieser mit Aufführungen wie Tänzen in der orchestra zu tun hat und stellt einen Bezug zu den auf die Bühne führenden Treppen in den klassischen Theatern her, die die Nutzung von

2I. G. Dufour „Le grand théâtre de Vendeuil-Caply (Oise) “, S. 107 sq.

22. J. Formigé, „L'autel aux Cygnes d'Arles et la thymélé dans les théâtres grécoromains".

23. Zu den Altären in Theatern vgl. die Überlegungen bei Ch. Mauduit et

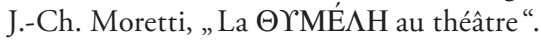




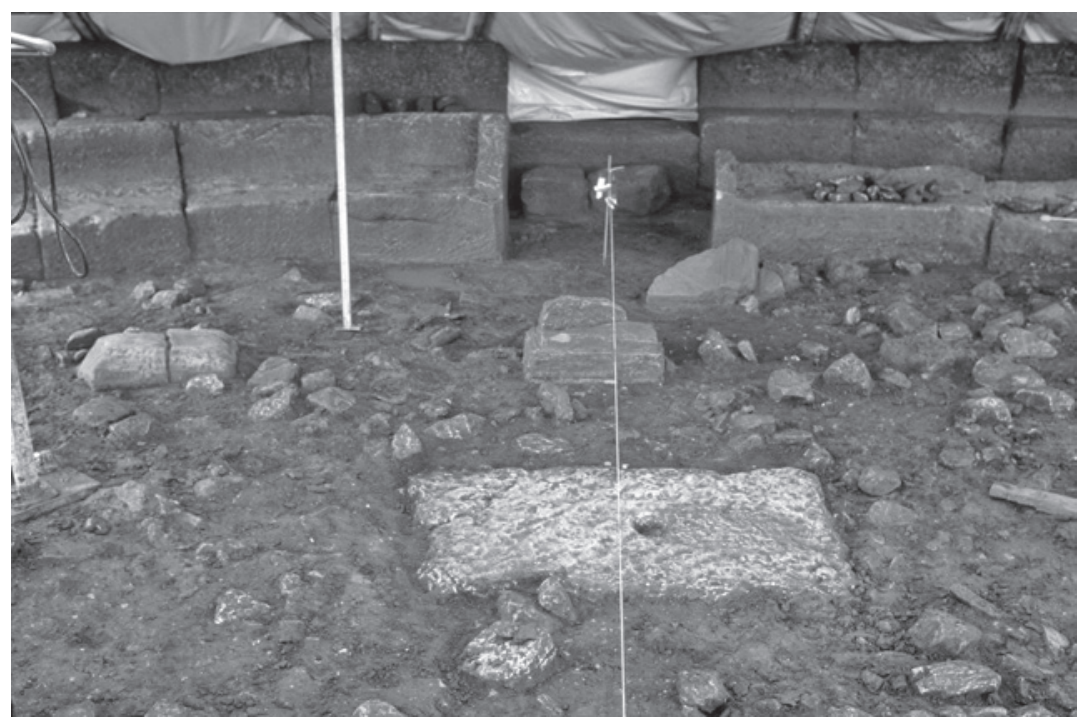

Abb. 11 — Fundamentreste zweier Altäre in der orchestra zwischen der ersten Sitzreihe und der Bühne.

Bühne und orchestra-Fläche während der Aufführung ermöglichen ${ }^{24}$. Auch zieht Jules Formigé für die Altäre den Standort für mobile Statuen in Betracht, die anlässlich eines Festes dort aufgestellt wurden ${ }^{25}$, was jedoch aufgrund der schlechten Erhaltung weder zu bestätigen noch zu widerlegen ist ${ }^{26}$.

In Vendeuil-Caply steht der Altar ${ }^{27}$ in der Mittelachse des Theaters zwischen der Bühne und den Zuschauerrängen vor dem sacellum ${ }^{28}$. Damit entspricht der Standort dem der Altäre von Dalheim. Gemeinsam mit den oben beschriebenen Eingängen neben dem Bühnengebäude und den beschriebenen Trittstufen wird deutlich, dass neben einem direkten Zugang in die orchestra die Bühne von dort problemlos betreten werden konnte und somit auch die Altäre sowohl von außerhalb durch die

24. Ibid., S. 23 sq.

25. Ibid., S. 34.

26. Vgl. hierzu auch die Thesen von G. Sauron, der den Altar im Zusammenhang mit dem Kaiserkult sieht: G. Sauron, „Les autels néo-attiques du théâtre d'Arles“, S. 213-216.

27. G. Dufour, „L'orchestra du grand théâtre de Vendeuil-Caply (Oise) et son sacellum", S. 154, Abb. 4.

28. Vgl. den Plan: Ibid., S. 149, Abb. 3. 


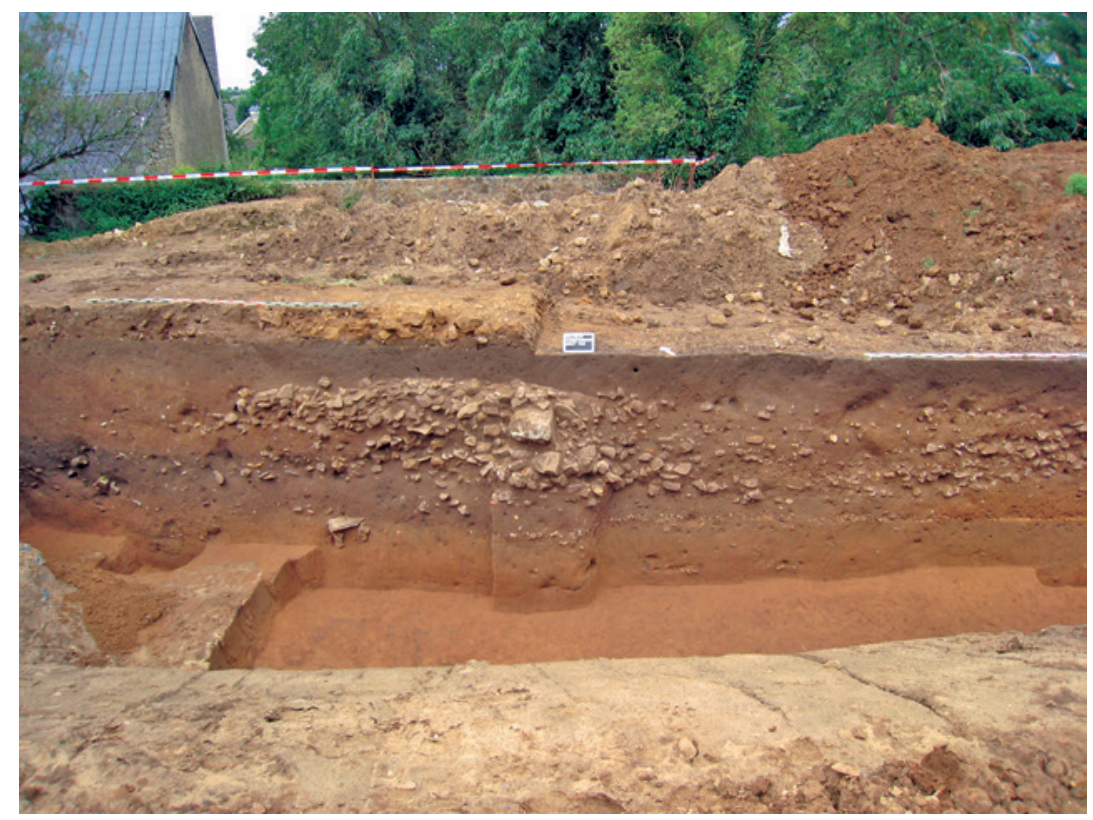

Abb. 12 - Profil durch die Straße um das Theater an der Nordwestseite des Theaters.

Eingänge als auch unmittelbar von der Bühne zu erreichen waren. Dies könnte auf eine funktionale Verknüpfung dieser drei Elemente hindeuten, die mit den von Elisabeth Bouley in Betracht gezogenen pompae in Verbindung zu bringen ist ${ }^{29}$. Dies würde für die Theater von VendeuilCaply und Dalheim bedeuten, dass diesen eine nicht genauer zu spezifizierende sakrale Funktion zukommt ${ }^{30}$. In dem Zusammenhang ist auch das in Vendeuil-Caply dokumentierte sacellum am Scheitelpunkt des orchestra-Bogens zu nennen. Durch diese sakralen Elemente wird auch die Funktion zumindest dieser Theater klar definiert ${ }^{31}$, ohne dabei Rückschlüsse auf alle gallorömischen Theater ziehen zu können ${ }^{32}$.

29. E. Bouley, „Les théâtres cultuels de Belgique et des Germanies “, S. 568.

30. Vgl. hierzu die Überlegungen von D. Tardy, dem Altar von Vendeuil-Caply eine nicht genau zu bestimmende rituelle Funktion zuzusprechen: D. Tardy, „Les lieux de culte dans les édifices de spectacle gallo-romains “.

31. Vgl. die Zusammenstellung bei Ibid., S. 546-548.

32. Zur sakralen Funktion der Theater im Zusammenhang mit den regelhaft in der direkten Nähe dokumentierten Tempel: Th. Lobüscher, Tempel-und Theaterbau in den Tres Galliae und den germanischen Provinzen, S. 13-20. 
Die Tatsache, dass nur in zwei gallorömischen Theatern Altäre in der orchestra nachgewiesen werden konnten, jedoch bei einer deutlich größeren Anzahl Eingänge neben dem Bühnengebäude und Trittstufen auf die Bühne erhalten sind, deutet darauf hin, dass mit einer ehemals deutlich größeren Anzahl an Altären gerechnet werden muss. Die Seltenheit dieser Altäre dürfte dadurch zu erklären sein, dass die Altäre im Zuge des Steinraubes als kompakte Steinblöcke sehr begehrt und auch leicht und rückstandslos zu entfernen waren. In Dalheim begünstigte das frühe Zusedimentieren der orchestra die Erhaltung der Altäre bzw. deren Fundamente.

\section{Das Ende des Theaters}

Das Ende der regulären Nutzung des Theaters ist in die Zeit nach den Germaneneinfällen der zweiten Hälfte des dritten Jahrhunderts zu datieren. Hiervon zeugen die Brandschichten im Inneren und direkt außerhalb des Südvomitoriums. Zudem wurde ein Großteil der Sitzsteine zum Bau des bereits erwähnten burgus verwendet. Von einer regelmäßigen Frequentierung während des vierten Jahrhunderts und bis ins frühe fünfte Jahrhundert zeugen mehr als 1000 Bronzemünzen, weitere Kleinfunde aus Bronze sowie große Mengen an Grobkeramik, die in der orchestra und zwischen den Sitzsteinen der ima cavea gefunden wurden, ohne dass beim momentanen Stand der Bearbeitung eine genaue funktionale Eingrenzung dieser Nutzung möglich ist ${ }^{33}$.

\section{Das Theaterumfeld}

Südlich des Theaters befand sich der oben bereits beschriebene mehr als $4 \mathrm{~m}$ tiefe Steinbruch mit treppenartigen, sehr steilen Wänden. Diesen verfüllte man bereits während der Nutzung des Theaters sukzessive mit verschiedenen Planierschichten (Abb. 3).

33. Ausgeschlossen werden kann eine fortifikatorische Funktion wie beispielsweise beim Theater von Avenches: G. Matter, „Die Sondierungen am römischen Theater, En Selley, Avenches 1998/1999“, S. 169-172. 
Zuunterst wurden stark sandige und mit Steinmetzschutt durchsetzte Sedimente ohne Funde dokumentiert, die während des Aushebens der Fundamentgräben für die cavea-Mauer angefallen sind und dann an der Theatersüdseite verfüllt wurden. Auf diesen Schichten liegen massive, insgesamt bis zu 1,80 m mächtige Schichtpakete, deren Grünfärbung gemeinsam mit zahlreichen Tierknochen- und Keramikfunden auf stark mit Fäkalien und Hausmüll durchsetzte Sedimente hindeutet. Dies ist zunächst verwunderlich, muss doch die Geruchsentwicklung direkt neben dem Südvomitorium erheblich gewesen sein. Doch finden sich für Müllgruben direkt bei Theater auch Beispiele in Augst und dem jüngst entdeckten Theater bei Kastel-Staadt ${ }^{34}$.

Als jüngste römische Schicht wurde ein mit Holzkohle und vielen, teilweise stark verbrannten Kleinfunden durchsetztes Sediment dokumentiert. Im Gegensatz zu den zuvor beschriebenen Schichten fanden sich hier neben großen Mengen an Keramikfunden auch zahlreiche Münzen, Bronze- und Glasobjekte - jedoch bedeutend weniger Tierknochen. Diese Zusammensetzung spricht für eine Deutung als Zerstörungsschicht, die anhand der Funde in die $270^{\mathrm{er}}$ Jahre zu datieren ist und folglich mit den Germaneneinfällen der zweiten Hälfte des dritten Jahrhunderts in Zusammenhang zu bringen ist. Hierbei handelt es sich um den Schutt des zerstörten vicus, der neben dem Theater an dem Steilhang entsorgt wurde.

Sowohl an der nordöstlichen als auch an der südöstlichen Außenseite des Theaters konnte eine parallel zur cavea-Mauer verlaufende, zweiphasige Straße dokumentiert werden, die nach einer ersten Durchsicht der Funde in einer der letzten Bauphasen des Theaters dort gebaut wurde und auch, wie geophysikalische Messungen gezeigt haben, weiter in Richtung Nordosten auf die Thermenanlage zuläuft (Abb. 12).

\section{Fazit und Ausblick}

Durch die mehrjährigen Grabungskampagnen im gallorömischen Theater von Dalheim sowie in dessen direktem Umfeld konnten

34. Augst: A. Furger, S. Deschler-Erb, Das Fundmaterial aus der Schichtenfolge beim Augster Theater, S. 137, Abb. 79-81; Kastel-Staadt: Freundliche Information B. Kremer (Rheinisches Landesmuseum Trier). 
wichtige neue Erkenntnisse zum Dalheimer Theater im Speziellen und zu gallorömischen Theatern im Allgemeinen gewonnen werden. Dies betrifft einerseits die neuen Informationen zu Nutzung des Areals vor dem Bau des Theaters als Steinbruch bzw. als Standort des nicht dem Theater zuzurechnenden Gebäudes. Andererseits ist durch den Nachweis der Straße eine wichtige Information zur infrastrukturellen Einbindung des Theaters in den vicus gegeben.

Die Auswertung der Grabungsergebnisse zeigt deutlich die Komplexität der Bauphasen. So können zwei Phasen des Einbaus hölzerner Sitzbänke unterschieden werden. Zusätzlich sind alle anderen Umbauten als präventive Maßnahmen zur Verhinderung von Bauschäden durch das zusätzlich aufgetragene Sediment in der summa cavea zu deuten.

Als letzter großer Eingriff in die Bausubstanz ist das Einbringen der Sitzreihen aus Kalkstein zu nennen. In der jüngsten Bauphase wurden dann die beiden vordersten Sitzbänke aus Sandstein sowie die Altäre eingebaut.

Durch die guten Erhaltungsbedingungen existieren erstmals für ein gallorömisches Theater eindeutige Hinweise auf die Prohedrie. Gemeinsam mit den erhaltenen Trittstufen auf die Bühne, den seitlichen Eingängen neben dem Bühnengebäude sowie den beiden Altären in der orchestra ergeben sich weiterhin wichtige Hinweise auf die Nutzung dieser Bereiche und letztendlich zur Funktion der gallorömischen Theater.

Peter Henrich

Deutsche Limeskommission

Bad Homburg 


\section{BIBLIOGRAPHIE}

Bonneau, Michel, „Le théâtre de Compierre “, Revue Archéologique de l'Est et du Centre-Est, 37 (1986), S. 130-136.

Bouley, Elisabeth, „Les théâtres cultuels de Belgique et des Germanies“, Latomus, 42 (1983), S. 546-571.

Delétang, Henri, „Le théâtre gallo-romain de Neung-sur-Beuvron (Loir-et-Cher) et son environnement ", Revue Archéologique du Centre, 27 (1988), S. 143-204.

Dufour, Gérard, „L'orchestra du grand théâtre de Vendeuil-Caply (Oise) et son sacellum ", Revue archéologique de la Picardie, 4 (1982), S. 145-158.

—, "Vendeuil-Caply “, Dossiers Histoire et Archéologie, 134 (1989), S. 69-72.

—, „Le grand théâtre de Vendeuil-Caply (Oise). Essai de restitution du sacellum et de la thymélé", in Spectacula II. Le théâtre antique et ses spectacles. Actes du colloque tenu au musée archéologique HenriPrades de Lattes les 27, 28, 29 et 30 avril 1989, éd. Christian Landes, Lattes, Musée archéologique de Lattes, 1992, S. 103-112.

Dumasy, Françoise, „Petit atlas des édifices de théâtre en Gaule romaine ", in Le goût du théâtre à Rome et en Gaule romaine, éd. Christian Landes, Lattes, Musée archéologique de Lattes, 1989, S. 43-75.

—, Le théâtre d'Argentomagus (Saint-Marcel, Indres), Paris, Maison des sciences de l'Homme, 2000 (Documents d'archéologie française 79).

—, „Les édifices de spectacle en Gaule du Nord. De la typologie à la chronologie ", in Les villes romaines du nord de la Gaule, éd. Roger Hanoune, Lille, Université de Lille, 2007, S. 447-465.

Fincker, Myriam, „Saint-Germain-d'Esteuil. Le théâtre. Analyse préliminaire des structures “, Aquitania, 17 (2000), S. 167-179. 
Fincker, Myriam, Thierry, François, „Nouvelles recherches sur le théâtre gallo-romain des Bouchauds (Charente) “, Aquitania, 3 (1985), S. 113-139.

Formigé, Jules, „L'autel aux Cygnes d'Arles et la thymélé dans les théâtres gréco-romains “, Revue Archéologique, 21 (1944), S. 21-24.

Furger, Alex, Deschler-Erb, Sabine, Das Fundmaterial aus der Schichtenfolge beim Augster Theater, Augst, Amt für Museen und Archäologie des Kantons Basel-Landschaft, 1992.

Gaston, Christophe, „Bâtiments , standardisés ‘ dans la pars rustica des villae: deux exemples récemment découverts en Franche-Comté", Revue Archéologique de l'Est, 57 (2008), S. 253-266.

Gillet, Evelyne, Demarez Léonce, Deroissart, Dominique, „Leuzeen-Hainaut/Blicquy: un édifice de spectacle gallo-romain sur le site du sanctuaire de la Ville d'Anderlecht", Chronique de l'Archéologie Wallonne, 4-5 (1996-1997), S. 23-26.

Huld-Zetsche, Ingeborg, Trierer Reliefsigillata Werkstatt I, Bonn, Römisch-Germanische Kommission des Deutschen Archäologischen Instituts, 1972.

Kenyon, Kathleen M., „, The Roman theatre at Verulamium, St.Albans “, Archaeologia, 84 (1934), S. 213-261.

Krier, Jean, „Le théâtre gallo-romain découvert en 1985 à Dalheim (Grand-Duché de Luxembourg) “, in Spectacula II. Le théâtre antique et ses spectacles. Actes du colloque tenu au musée archéologique Henri-Prades de Lattes les 27, 28, 29 et 30 avril 1989, éd. Christian Landes, Lattes, Musée archéologique de Lattes, 1992, S. 121-132.

—, „Das Thermengebäude des römischen Vicus von Dalheim? “, Musée Info, 18 (2005), S. 61-63.

—, "Agglomération romaine de Dalheim-Ricciacum", in Ricciacus. 30 joer Ricciacus Frenn, hrsg. von Ricciacus Frenn Asbl., Luxembourg, Imprimerie Centrale Luxembourg, 2007, S. 23-39.

Krier, Jean, Wagner, Robert, „Das Theater des römischen Vicus in Dalheim (Luxemburg) “, Archäologisches Korrespondenzblatt, 15 (1985), S. 481-495.

—, „Das römische Theater in Dalheim “, Hémecht, 37 (1985), S. 587 614. 
La Croix, Camille de, „Etude sur le théâtre gallo-romain des Bouchauds (Charente) et son déblaiement ", Bulletin de la société archéologique et historique de la Charente, 8 (1908), S. 65-172.

Loвüscher, Thomas, Tempel- und Theaterbau in den Tres Galliae und den germanischen Provinzen, Rhaden, Verlag Marie Leidorf, 2002.

Matter, Georg, „, Die Sondierungen am römischen Theater, En Selley, Avenches 1998/1999“, Bulletin de l'Association Pro Aventico, 41 (1999), S. 147-198.

Mauduit, Christine, Moretti, Jean-Charles, „La OrMÉ $\Lambda \mathrm{H}$ au théâtre ", in Fronts de scène et lieux de culte dans le théâtre antique, éd. Jean-Charles Moretti, Lyon, Maison de l'Orient et de la Méditerranée, 2009, S. 11-21 (TMO 52).

Olivier, Albéric, „Grand“, Dossiers Histoire et Archéologie, 116 (1987), p. $72-77$.

—, „Autun: Esquisse d'une restitution architecturale “, Dossiers Histoire et Archéologie, 134 (1989), S. 36-40.

Olivier, Albéric, Rabeisen, Elisabeth, „Le théâtre d'Alésia “, Dossiers Histoire et Archéologie, 134 (1989), S. 58-63.

Olivier, Lucien, „Arleuf“, Dossiers Histoire et Archéologie, 134 (1989), S. 84-85.

—, "Le théâtre antique de Bardiaux “, in Spectacula II. Le théâtre antique et ses spectacles. Actes du colloque tenu au musée archéologique Henri-Prades de Lattes les 27, 28, 29 et 30 avril 1989, éd. Christian Landes, Lattes, Musée archéologique de Lattes, 1992, S. 57-62.

Petit, Jean-Paul, „L'architecture privée dans l'agglomération secondaire de Bliesbruck. Bilan et perspectives de recherche ", in Festschrift Jean Schaub, éds Jean-Michel Massing, Jean-Paul Petit, Paris, Serpenoise, 1993, S. 129-160.

Petit, Jean-Paul, Mangin, Michel, Brunella, Patrick, Atlas des agglomérations secondaires de la Gaule Belgique et des Germanies, Paris, Errance, 1994.

Renaud, Jean, „Le théâtre mixte d'Aquae Segetae“, Bulletin de la Diana, 36 (1960), S. 281-302.

Sauron, Gilles, „Les autels néo-attiques du théatre d'Arles“, in L'espace sacrificiel dans les civilisations méditerranéenes de l'Antiquité. Actes du colloque tenu à la Maison de l'Orient, Lyon, 4-7 juin 1988, éds 
Roland Etienne, Marie-Thérèse Le Dinahet, Paris, Boccard, 1991, S. 205-216.

Tardy, Dominique, „Les lieux de culte dans les édifices de spectacle gallo-romains ", in Fronts de scène et lieux de culte dans le théatre antique, éd. Jean-Charles Moretti, Lyon, Maison de l'Orient et de la Méditerranée, 2009, S. 175-188 (TMO 52).

Wagner, Robert, „Théâtre gallo-romain de Dalheim “, Musée Info, 13 (2000), S. 32-35.

Photographische Krediten

Abb. 1 und 2:

MNHA.

Abb. 3, 4, 6-7, 10 und 12:

MNHA P. Henrich.

Abb. 5:

MNHA M. Diederich.

Abb. 8, 9 und 11:

MNHA R. Wagner. 
